\title{
A three-generation family with metaphyseal dysplasia, maxillary hypoplasia and brachydactyly (MDMHB) due to intragenic RUNX2 duplication
}

\author{
Amina Al-Yassin ${ }^{1} \cdot$ Alistair D. Calder ${ }^{2} \cdot$ Mike Harrison $^{3} \cdot$ Tracy Lester $^{4} \cdot$ Helen Lord $^{4} \cdot$ Michael Oldridge $^{4}$. \\ Sophie Watkins ${ }^{3} \cdot$ Richard Keen $^{5} \cdot$ Emma L. Wakeling $^{1}$
}

Received: 26 September 2017 / Revised: 29 March 2018 / Accepted: 11 April 2018 / Published online: 11 June 2018

(c) European Society of Human Genetics 2018

\begin{abstract}
Metaphyseal dysplasia with maxillary hypoplasia and brachydactyly (MDMHB) is an autosomal-dominant skeletal dysplasia characterised by metaphyseal flaring of the long bones, enlargement of the medial halves of the clavicles, maxillary hypoplasia, brachydactyly, dental anomalies and mild osteoporosis. To date, only one large French Canadian family and a Finnish woman have been reported with the condition. In both, intragenic duplication encompassing exons 3-5 of the RUNX2 gene was identified. We describe a new, three-generation family with clinical features of MDMHB and an intragenic tandem duplication of RUNX2 exons 3-6. Dental problems were the primary presenting feature in all four affected individuals. We compare the features in our family to those previously reported in MDMHB, review the natural history of this condition and highlight the importance of considering an underlying skeletal dysplasia in patients presenting with significant dental problems and other suggestive features, including disproportionate short stature and/or digital anomalies.
\end{abstract}

\section{Introduction}

Metaphyseal dysplasia with maxillary hypoplasia and brachydactyly (MDMHB; OMIM 156510) was first reported in 1982 by Halal et al. in four generations of a large French Canadian family [1]. The authors described an autosomal-dominant disorder characterised by metaphyseal flaring of the long bones, enlargement of the medial halves of the clavicles, maxillary hypoplasia,

Emma L. Wakeling

e.wakeling@nhs.net

1 North West Thames Regional Genetic Service, London North West Hospitals NHS Trust, Harrow, Middlesex HA1 3UJ, UK

2 Department of Radiology, Great Ormond Street Hospital for Children NHS Foundation Trust, London WC1N 3JH, UK

3 Guy's Dental Hospital, Guy's Tower, Great Maze Pond, London SE1 9RT, UK

4 The Oxford Medical Genetics Laboratories, Churchill Hospital, Oxford OX3 7LJ, UK

5 Royal National Orthopaedic Hospital, Brockley Hill, Stanmore HA7 4LP, UK brachydactyly, dystrophic yellow teeth and distinctive facial features. In addition, X-rays showed thin diaphyseal cortex in long bones, platyspondyly and mild osteoporosis. More recently, several individuals from a family with a strikingly similar phenotype and originating from the same region of Quebec were shown to have intragenic duplication of exons 3-5 of the Runt-related transcription factor 2 (RUNX2) gene [2]. Functional studies showed that this duplication resulted in a gain of function [2]. A similar, though not identical, in-frame duplication of exons 3-5 of RUNX2 was subsequently reported in a 20 -year-old Finnish woman with clinical and radiological signs of MDMHB [3].

We describe a three-generation family with typical clinical and radiological features of MDMHB. Intragenic duplication of $R U N X 2$ exons 3-6 was found in three affected individuals. This report provides further information regarding the variability of the clinical phenotype and natural history of this rare condition. We also highlight the importance of considering an underlying skeletal dysplasia in patients presenting with significant dental problems and other suggestive features, including disproportionate short stature and digital anomalies. 

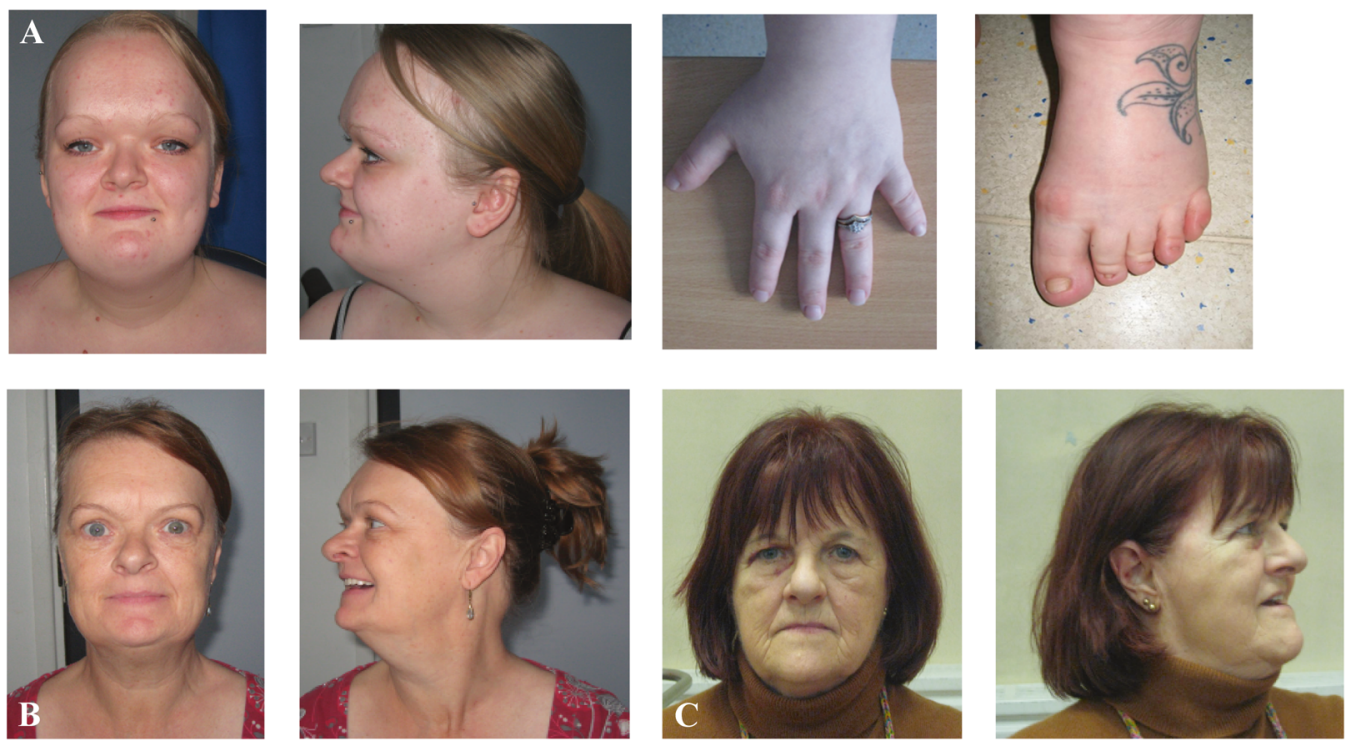

Fig. 1 Clinical features in the proband (a), her mother (b) and grandmother (c). Maxillary hypoplasia can be seen in all three individuals; the proband and her mother both have hypertelorism; brachydactyly is present in the proband

\section{Subjects and methods}

The proband is a 28 -year-old female who was first referred to the genetic service aged 2 years with yellow, dysplastic teeth and a family history of similar dental anomalies. She also had delayed closure of her anterior fontanelle, recurrent otitis media and a squint. At the time, the family were given a diagnosis of autosomal-dominant amelogenesis imperfecta.

She re-presented to clinical genetics during pregnancy. She had widespread dental caries and oligodontia. Her height was on the 0.4th centile and occipito-frontal circumference (OFC) on 25th-50th centile. She had a broad forehead, flat nasal bridge, mild hypertelorism and maxillary hypoplasia (Fig. 1a). She also had brachydactyly with proximally placed, short thumbs and fifth finger clinodactyly bilaterally. Her feet were small with hypoplastic, deep-set second-fifth toenails. There was genu valgum.

The proband's mother, aged 53 years, had late eruption of both primary and secondary teeth, which were small and yellowing. She has worn dentures since the age of 17 years, following full dental extraction. She fractured her right foot at 30 years following trauma. Her height is on ninth centile. Her facial features are similar, with maxillary hypoplasia (Fig. 1b). She has genu valgum but normal hands and feet.

The maternal uncle also had discoloured teeth and has also worn dentures since the age of 17 years. His features are reported to be milder, with larger, less eroded teeth prior to extraction, height on 50th centile and normal hands and feet.

The maternal grandmother, aged 77 years, had a heart murmur in childhood (which has since resolved) and delayed closure of her anterior fontanelle. She also had late eruption of her teeth, which were small and yellowing. She has worn dentures since the age of 17 years. She had one fracture of her wrist, following an accident. Her height is on second centile and OFC on 9th-25th centile. Her facial features are similar, with maxillary hypoplasia (Fig. 1c), but her hands and feet are normal.

\section{Radiological imaging}

Skeletal survey in the proband (Fig. 2) demonstrated broadening of the clavicles, particularly medially, mild rib broadening, osteopenia with multiple vertebral compression fractures and brachyphalangy of the distal phalanges of all digits, particularly the thumbs, and middle phalanges of the index and little fingers. There was also undermodelling of the long bones, particularly the lower femora, prominent supraorbital ridging of the skull and shortening of the left fifth metacarpal.

The mother and grandmother both had clavicular broadening on chest radiographs but no evidence of brachyphalangy on hand radiographs. The grandmother, but not the mother, had mild broadening of the ribs.

\section{Dental investigation}

The proband had multiple dental anomalies, including a widespread enamel hypomaturation defect, resulting in varying degrees of intrinsic pigmentation. Unusual vertical cracks were apparent in the labial surfaces of the maxillary 

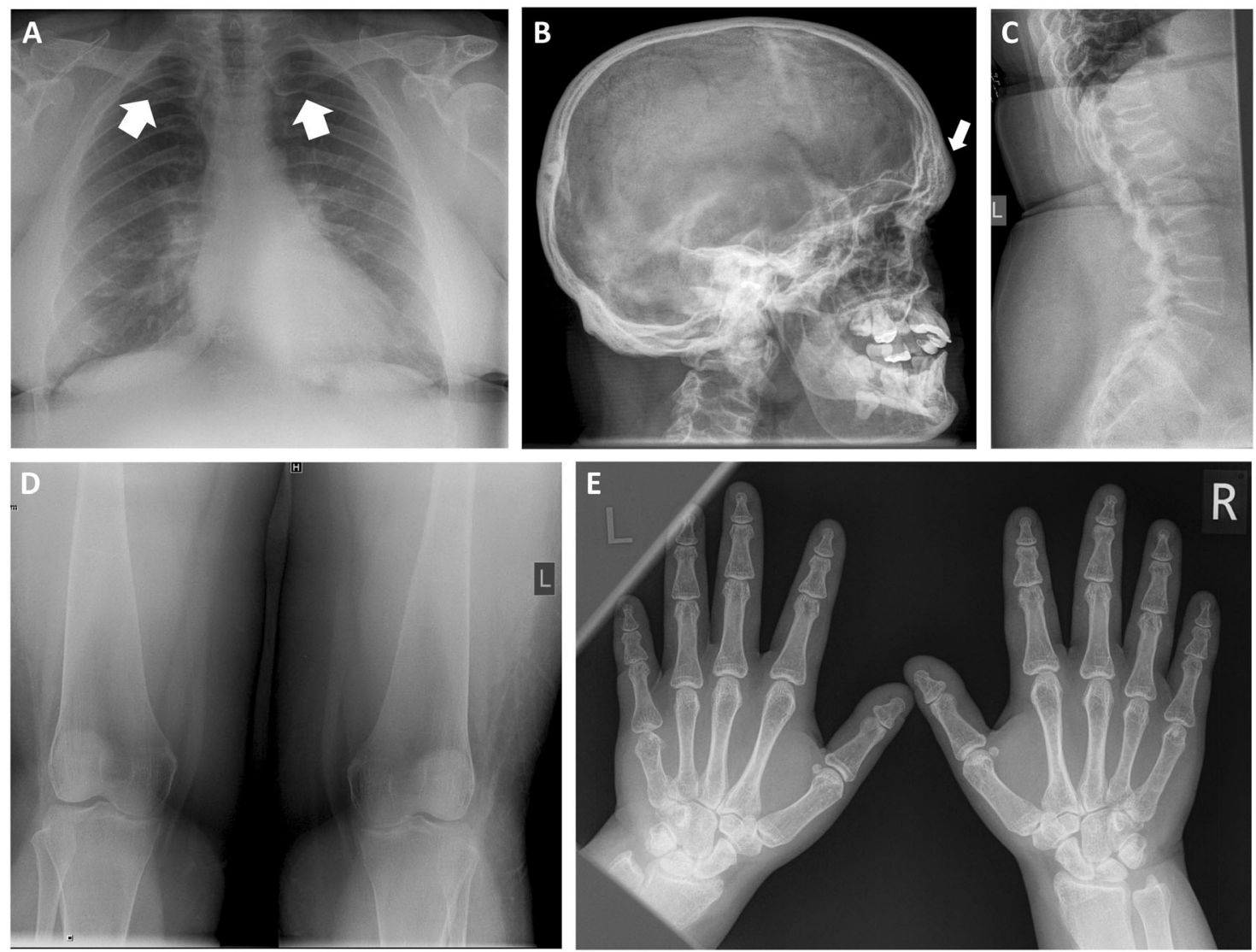

Fig. 2 Radiographic findings in proband, aged 27 years. a Posteroanterior chest radiograph. The clavicles are broadened, particularly medially (arrows), with mild broadening of the ribs. b Lateral skull radiograph. There is supraorbital ridging (arrow), an unusual finding in a female. The posterior skull vault is thickened with a prominent external occipital protuberance. Multiple dental crowns are noted. c Lateral lumbar spine radiograph. There are compression fractures of
T11, L1, L2 and L3. There is an osteopenic appearance. d Anteroposterior radiograph of both knees. There is undermodelling of the lower femoral metaphyses. e Dorso-palmar radiograph of both hands. There is moderate shortening of the distal phalanges of both thumbs and the middle phalanges of the index and little fingers bilaterally There is milder shortening of the distal phalanges of the index through little fingers. The left fifth metacarpal is also short incisors, and there was a particularly complex fissure pattern on the occlusal surfaces of the molar teeth (Fig. 3a, b).

The jaw radiograph (Fig. 3c) showed failure of eruption of multiple teeth, oligodontia of two maxillary premolars, sclerosed pulp chambers, blunted roots and areas of irregular sclerotic cancellous bone in the mandible. The maxillary sinuses appeared to be either totally obliterated or developmentally absent, consistent with maxillary hypoplasia.

\section{Bone investigations}

Dual-energy X-ray absorptiometric (DEXA) scan in the proband revealed reduced bone density at the right hip with a $Z$-score of -2.3 . Her Vitamin D level was $43 \mathrm{nmol} / \mathrm{L}$ and supplementation was recommended. The rest of the bone and renal profile was normal. DEXA scans in both the mother and grandmother were normal for age, with no evidence of osteoporosis.

\section{Genetic testing}

Blood samples were taken from the proband, mother and grandmother with informed consent. Multiplex ligationdependent probe amplification (MLPA) was carried out according to the manufacturer's recommendations using the MRC Holland MLPA Kit P080-C1 containing probes to coding exons of RUNX2, a 3730 automated analyser (ABI) and Coffalyser software (MRC Holland). An intragenic duplication encompassing exons 3-6 of RUNX2 (HGVS nomenclature: c. $(58+1$ _59-269)_(859+1_860-1)dup) was identified in all three individuals. (Reference sequence NM_001024630.3; exons numbered sequentially with the first coding exon being exon 2) [2].

In order to determine whether the duplication was in tandem as expected, RNA was extracted from blood taken into PAXgene blood RNA tubes using the PAXgene Blood RNA Kit according to the manufacturer's protocol (PreAnalytiX). cDNA was made using the Invitrogen 
Fig. 3 Dental findings in the proband. a Dental frontal view. b Mandibular arch view. c Dental pantomogram


Dup exons 3-6
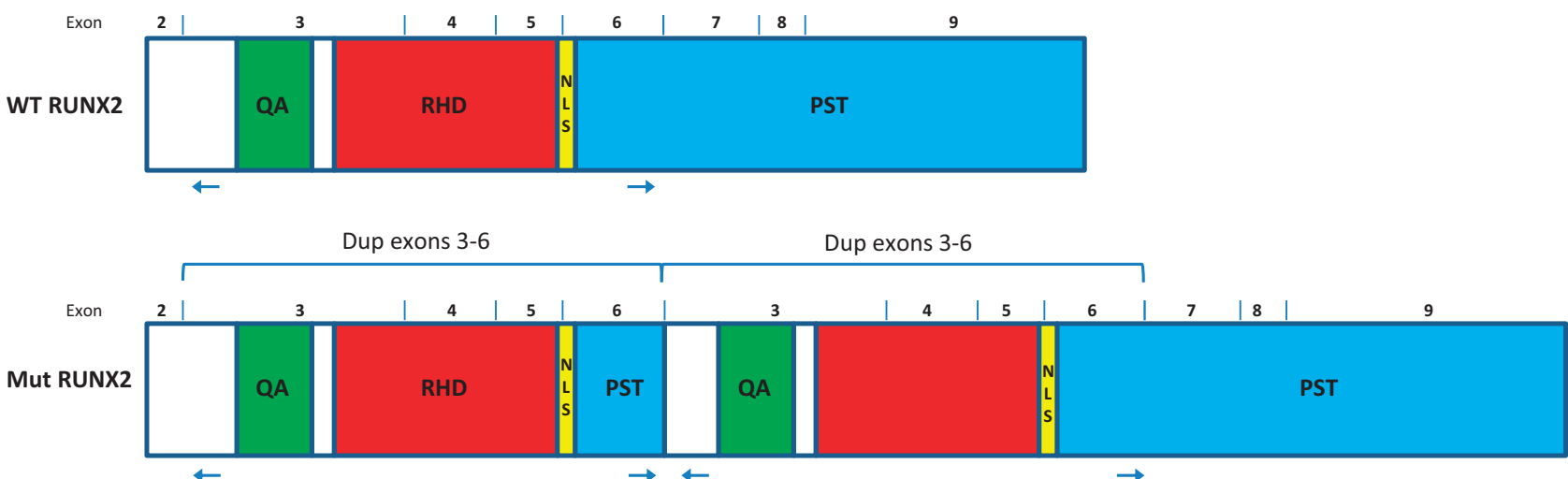

Fig. 4 Schematic of 521 amino acid wild-type RUNX2 protein (upper panel) and predicted mutant protein after duplication of exons 3-6 (lower panel). Numbers above the domain structures show the coding exons 2-9. Brackets show the duplicated region. Arrows below show

ThermoScript Reverse Transcription Kit according to the manufacturer's random hexamer protocol. Two sets of PCR primers were designed that would only amplify cDNA from the affected individuals if the duplication is in tandem, with forward primers in exon 6 and reverse primers in exon 3 (Fig. 4). A product of the predicted size was seen for all three affected individuals but was absent in the normal control sample for both primer sets. POLRID control primers were used to confirm the presence of cDNA in the control sample. PCR products were then sequenced, demonstrating that exon 3 is spliced to exon 6 and that this is a tandem duplication, which is predicted to be in-frame.

Information regarding the variant and phenotype has been submitted to the DECIPHER database as open access (https://decipher.sanger.ac.uk/patient/362320\#overview/pa tient-general). the position of primers designed to PCR across the duplication (exon 6/3). Domains shown: QA poly Glu/poly Ala domain, RHD Runt homology domain, NLS nuclear localisation signal, PST proline-/ serine-/threonine-rich domain

\section{Discussion}

$R U N X 2$ (OMIM 600211) is a member of the $R U N X$ family of transcription factors and encodes a nuclear protein, which is a key regulator of osteoblast and terminal chondrocyte differentiation, essential for bone formation and mineralisation [4]. RUNX2 regulates a wide range of bone-related genes and has been implicated in many different signalling pathways. These include transforming growth factor- $\beta$, bone morphogenetic protein, fibroblast growth factor and Wnt signalling pathways, all of which have also been implicated in tooth morphogenesis [5]. Runx2-deficient mice have complete absence of mature osteoblasts and ossification [6, 7]; molar development is arrested at early cap stage [8]. Paradoxically, triple transgenic mice overexpressing Runx 2 have decreased body size, osteopenia and 
Table 1 Clinical and radiological features in patients reported with MDMHB

\begin{tabular}{|c|c|c|c|c|c|c|c|}
\hline & \multicolumn{3}{|c|}{ Current report } & \multirow{2}{*}{$\begin{array}{l}\text { Avela et al. } \\
\text { (2014) [3] }\end{array}$} & \multirow{2}{*}{$\frac{{ }^{\mathrm{a}} \text { Moffatt et al. (2013) [2] }}{6 \text { individuals }}$} & \multicolumn{2}{|c|}{ Halal et al. (1982) [1] } \\
\hline & Proband & Mother & Grandmother & & & Proband (V-3) & Mother (IV-9) \\
\hline Age at report (years) & 28 & 53 & 77 & 20 & $14,15,16,17,41,50$ & 4 & 29 \\
\hline Dystrophic yellowish teeth & + & + & + & + & $6 / 6$ & + & + \\
\hline Maxillary hypoplasia & + & + & + & + & $5 / 6$ & + & + \\
\hline $\begin{array}{l}\text { Delayed closure of anterior } \\
\text { fontanelle }\end{array}$ & + & - & + & NR & NR & NR & NR \\
\hline Height (centile) & 0.4 th & 9th & 2nd & $3 \mathrm{rd}$ & $\begin{array}{l}<5 \text { th, } 10 \text { th, } 25 \text { th, }<5 \text { th } \\
<5 \text { th, 5th }\end{array}$ & 50th-75th & $3 \mathrm{rd}$ \\
\hline Metaphyseal dysplasia & + & + & + & + & $2 / 2$ & + & + \\
\hline Osteoporosis & + & N/A & N/A & + & Compression fractures in 3 & + & + \\
\hline Brachydactyly & + & - & - & + & $0 / 2$ & - & + \\
\hline Clavicular broadening & + & + & + & + & $2 / 2$ & + & + \\
\hline
\end{tabular}

+ present, - absent, $N R$ not reported

${ }^{a}$ Moffatt et al. described clinical features in detail in six patients; full skeletal survey was carried out in two of these cases [2]. The frequency of individual features in this family is recorded as a proportion of those for whom information is available

diminished osteogenesis [9]. It has been postulated that complete maturation of immature osteoblasts requires $R U N X 2$ suppression during osteoblast differentiation.

Intragenic duplication encompassing exons $3-5$ of the $R U N X 2$ gene was previously reported in a French Canadian family with MDMHB and one other, unrelated case from Finland [2,3]. An intragenic duplication encompassing exons $3-5$ of the $R U N X 2$ gene was previously reported in a French Canadian family with MDMHB and one other, unrelated case from Finland [2, 3]. Moffatt et al. carried out transfection studies with mouse Runx2 cDNA, demonstrating markedly higher cellular levels of mutated, as compared to wild-type, RUNX2 [2]. They also showed an increase in transactivation activity for the mutant RUNX2 compared to wild-type. These observations suggest that MDMHB results from a gain of function of $R U N X 2$ due to increased cellular levels of mutant protein and/or an increase in transactivation activity of the mutant protein. Our patient also has duplication of the functionally important QA and RUNT domains (exons 3-5), along with the nuclear localisation signal and part of the PST domain (exon 6) (Fig. 4). Unlike the exon 3-5 duplication reported by Moffatt et al., the variant in our patient does not lead to a change of amino acid at the breakpoint. However, the strikingly similar phenotype in our family, as compared to the other reported cases of intragenic duplication, suggests that the functional consequences are the same. In contrast, increased copy number associated with whole gene duplication of RUNX2 leads to a different, distinct phenotype, with metopic craniosynostosis [10]. It may be that alternative mechanisms, such as disruption of regulatory elements, are playing a role in the phenotype in these cases. Overall, our findings provide further evidence that intragenic tandem, in-frame duplications of RUNX2 are the cause of MDMHB, consistent with a gain-of-function mechanism due to possible overexpression of the gene.

The clinical findings in our family, together with those in previously reported patients with MDMHB are summarised in (Table 1) [1-3]. Dental anomalies are a consistent finding and are usually the presenting feature. Specific skeletal findings (metaphyseal dysplasia, maxillary hypoplasia, clavicular broadening and mild osteoporosis) are also seen in all/almost all cases. However, other features are highly variable. Although some affected individuals have final height below third centile, in others this is well within the normal range, albeit usually $<50$ th centile. Brachydactyly is only present in a minority ( $3 / 8$ reported cases). Delayed closure of the fontanelle was present in two of the cases reported here. Although not described before in association with MDMHB, this may in part be due to the lack of retrospective clinical data available for individuals previously reported.

Mild osteoporosis was described in the original family reported and has also been found in other affected individuals, including our proband $[1,2]$. This finding is consistent with the observation of osteopenia in transgenic mice overexpressing Runx [9]. Patients with MDMHB do not appear to be at significant risk of recurrent, unexplained fractures of long bones, even with increasing age. Back pain should, however, prompt further investigation to rule out spinal compression fractures.

A $1.1 \mathrm{Mb}$ duplication encompassing RUNX2 was identified in two affected cousins with metopic synostosis and hypodontia [10]. Both intervening obligate carriers were also reported to have hypodontia; no other clinical features were mentioned. This report provides further evidence of consistent dental problems, with otherwise variable intrafamilial phenotype, associated with $R U N X 2$ duplication. 
Heterozygous loss-of-function RUNX2 variants are associated with cleidocranial dysplasia (CCD; OMIM 119600) [11]. This condition is characterised by absent, or severely hypoplastic, clavicles and delayed closure of the anterior fontanelle. The majority of affected individuals also have dental abnormalities, with supernumerary permanent teeth. Other radiological features include hypomineralisation of the skull vault, Wormian bones, a narrow thorax, hypoplasia of the iliac wings and absent/delayed ossification of the pubic bones. In many respects, the two conditions present with opposite phenotypes. In MDMHB, there is widening of the clavicles, thickening of the skull vault and hypodontia with small, yellow dystrophic teeth. However, given the evidence for RUNX2 gain of function in MDMHB, it is interesting to note that there is also overlap in skeletal and dental features. Presence of Wormian bones, delayed closure of the anterior fontanelle, short distal phalanges, hypertelorism, genu valgum and delayed dental eruption have all been reported in both CCD and MDMHB.

Enamel defects have been described in association with a number of skeletal dysplasias, which should be considered in the differential diagnosis of MDMHB. Weyers acrofacial dysostosis (OMIM 193530) is characterised by enamel hypoplasia, nail dystrophy, postaxial polydactyly and mild short stature [12]. The condition is associated with dominant variants in EVC2 and EVC and is allelic to Ellis-van Creveld syndrome. Tricho-dento-osseous syndrome (OMIM 190320), caused by autosomal-dominant variants in $D L X 3$, is associated with enamel hypoplasia, mild osteosclerosis, brittle nails and blond, dry, curly hair [13]. Autosomal-recessive variants in LTBP3 have been described in association with amelogenesis imperfecta, short stature and platyspondyly (OMIM 601216) [14]. Patients with non-lethal Raine syndrome (OMIM 259775), due to autosomal-recessive FAM20C variants, also have amelogenesis imperfecta and severe maxillary hypoplasia in association with intracranial calcification, low phosphate level and abnormal bone density (with both sclerosis, particularly of the skull base, and osteoporosis) [15].

The findings in this three-generation family, and other cases, demonstrate the phenotypic variability of MDMHB, even within one individual family. Dental anomalies and skeletal findings (metaphyseal dysplasia, clavicular broadening and osteoporosis) are the most consistent features. However, the associated reduction in bone density appears to have relatively mild/no clinical effect, even in older age. The diagnosis may therefore be easily missed in individuals presenting mainly with dental problems. This report illustrates the importance of considering an underlying skeletal dysplasia in patients with dental anomalies and other suggestive features (including short stature, distinctive facial features and digital anomalies), either in themselves and/or other close relatives.

Acknowledgements We thank Professor Andrew Wilke, Professor of Genetics at the University of Oxford, for his advice regarding the functional significance of the RUNX2 intragenic duplication identified in our family.

\section{Compliance with ethical standards}

Conflict of interest The authors declare that they have no conflict of interest.

\section{References}

1. Halal F, Picard JL, Raymond-Tremblay D, de Bosset P, Opitz JM. Metaphyseal dysplasia with maxillary hypoplasia and brachydactyly. Am J Med Genet. 1982;13:71-9.

2. Moffatt P, Amor MB, Glorieux FH, et al. Metaphyseal dysplasia with maxillary hypoplasia and brachydactyly is caused by a duplication in RUNX2. Am J Hum Genet. 2013;92:252-8.

3. Avela K, Hirvinen H, Amor MB, Rauch F. Metaphyseal dysplasia with maxillary hypoplasia and brachydactyly in a Finnish woman: first confirmation of a duplication in RUNX2 as pathogenic variant. Eur J Med Genet. 2014;57:617-20.

4. Stein GS, Lain JB, van Wijen AJ, et al. Runx2 control of organization, assembly and activity of the regulatory machinery for skeletal gene expression. Oncogene. 2004;23:4315-29.

5. Zhang YD, Chen Z, Song YQ, Liu C, Chen YP. Making a tooth: growth factors, transcription factors and stem cells. Cell Res. 2005;15:301-16.

6. Komori T, Yagi H, Nomura S, et al. Targeted disruption of Cfba1 results in a complete lack of bone formation owing to maturational arrest of osteoblasts. Cell. 1997;89:755-64.

7. Otto F, Thornell AP, Crompton T, et al. Cfba1, a candidate gene for cleidocranial dysplasia syndrome, is essential for osteoblast differentiation and bone development. Cell. 1997;89:765-71.

8. D'Souza RN, Aberg T, Gaikwad J, et al. Cfbal is required for epithelial-mesenchymal interactions regulating tooth development in mice. Development. 1999;126:2911-20.

9. He N, Xiao Z, Yin T, et al. Inducible expression of Runx2 results in multiorgan abnormalities in mice. J Cell Bichem. 2011;112:653-65.

10. Mefford HC, Shafer N, Antonacci F, et al. Copy number variation analysis in single-suture craniosynostosis: multiple rare variants including RUNX2 duplication in two cousins with metopic craniosynostosis. Am J Med Genet. 2010;152A:2203-10.

11. Mundlos S, Otto F, Mundlos C, et al. Mutations involving the transcription factor CBFA1 cause cleidocranial dysplasia. Cell. 1997;89:773-9.

12. Ye X, Song G, Fan M, et al. A novel heterozygous deletion in the EVC2 gene causes Weyers acrofacial dysostosis. Hum Genet. 2006;119:199-205.

13. Price JA, Bowden DW, Wright JT, Pettenati MJ, Hart TC. Identification of a mutation in DLX3 associated with tricho-dentoosseous (TDO) syndrome. Hum Mol Genet. 1998;7:563-9.

14. Verloes A, Jamblin P, Koulischer L, Bourguignon JP. A new form of skeletal dysplasia with amelogenesis imperfecta and platyspondyly. Clin Genet. 1996;49:2-5.

15. Elalaoui SC, Al-Shegaih N, Ratbi I, et al. Non lethal Raine syndrome and differential diagnosis. Eur J Med Genet. 2016;59:577-83. 